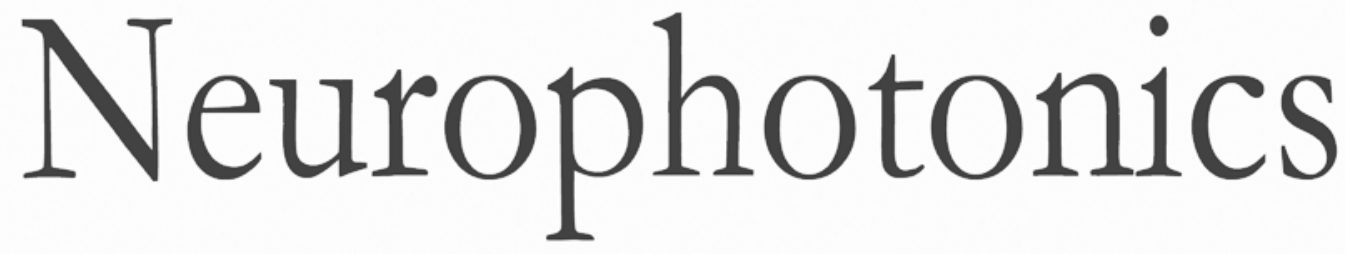

\title{
Superresolving dendritic spine morphology with STED microscopy under holographic photostimulation
}

\author{
Marcel Andreas Lauterbach \\ Marc Guillon \\ Claire Desnos \\ Dany Khamsing \\ Zahra Jaffal \\ François Darchen \\ Valentina Emiliani
}




\title{
Superresolving dendritic spine morphology with STED microscopy under holographic photostimulation
}

\author{
Marcel Andreas Lauterbach, ${ }^{a, \dagger}$ Marc Guillon, ${ }^{a}$ Claire Desnos, ${ }^{b}$ Dany Khamsing, ${ }^{b}$ Zahra Jaffal, ${ }^{b}$ \\ François Darchen, ${ }^{\mathrm{b}}$ and Valentina Emiliani, ${ }^{\mathrm{a}, *}$ \\ aUniversity Paris Descartes, Wavefront-Engineering Microscopy Group, Neurophotonics Laboratory, CNRS UMR8250, Sorbonne Paris Cité, \\ 45, rue des Saints Pères, Paris 75006, France \\ bUniversity Paris Descartes, Synapic Trafficking Group, Neurophotonics Laboratory, CNRS UMR8250, Sorbonne Paris Cité, 45, \\ rue des Saints Pères, Paris 75006, France
}

\begin{abstract}
Emerging all-optical methods provide unique possibilities for noninvasive studies of physiological processes at the cellular and subcellular scale. On the one hand, superresolution microscopy enables observation of living samples with nanometer resolution. On the other hand, light can be used to stimulate cells due to the advent of optogenetics and photolyzable neurotransmitters. To exploit the full potential of optical stimulation, light must be delivered to specific cells or even parts of cells such as dendritic spines. This can be achieved with computer generated holography $(\mathrm{CGH})$, which shapes light to arbitrary patterns by phase-only modulation. We demonstrate here in detail how CGH can be incorporated into a stimulated emission depletion (STED) microscope for photostimulation of neurons and monitoring of nanoscale morphological changes. We implement an original optical system to allow simultaneous holographic photostimulation and superresolution STED imaging. We present how synapses can be clearly visualized in live cells using membrane stains either with lipophilic organic dyes or with fluorescent proteins. We demonstrate the capabilities of this microscope to precisely monitor morphological changes of dendritic spines after stimulation. These all-optical methods for cell stimulation and monitoring are expected to spread to various fields of biological research in neuroscience and beyond. $\odot$ The Authors. Published by SPIE under a Creative Commons Attribution 3.0 Unported License. Distribution or reproduction of this work in whole or in part requires full attribution of the original publication, including its DOI. [DOI: 10.1117/1.NPh.3.4.041806]
\end{abstract}

Keywords: STED; STED microscopy; holography; computer generated holography; spine morphology; plasticity; photostimulation; uncaging.

Paper 16014SSR received Mar. 10, 2016; accepted for publication May 31, 2016; published online Jun. 22, 2016.

\section{Introduction}

Optical developments of the last years provide unique possibilities for the observation and active control of physiological processes at the cellular and subcellular scale.

Recordings with high spatial resolution at physiological conditions can be provided by optical superresolution methods. ${ }^{2,3}$ stimulated emission depletion (STED) microscopy ${ }^{2,4}$ achieves spatial resolution in the nanometer range $\mathrm{e}^{5,6}$ and temporal resolution up to the millisecond range. ${ }^{7-10}$ Due to this high time resolution and compatibility with living thick tissues, it is a valuable tool for functional imaging in neuroscience.

The ability to observe neurons at the subcellular scale of dendrites, axons, and synapses is critical for understanding neuronal function. Neurons form connections for intercellular signal transmission at synapses. The postsynaptic site of most excitatory synapses is formed by spines, which are protrusions from dendrites with dimensions varying from below $100 \mathrm{~nm}$ for their neck diameter up to a few microns for total length. The morphology of these spines plays an essential role for the synaptic function, ${ }^{11,12}$ for synaptic plasticity, ${ }^{13,14}$ and, more generally, for brain development. ${ }^{15}$ It was found that the size of spines

*Address all correspondence to: Valentina Emiliani, E-mail: valentina.emiliani@ parisdescartes.fr

${ }^{\dagger}$ Current address: Max Planck Institute for Brain Research, Department of Neural Systems, Max-von-Laue-Str. 4, 60438 Frankfurt am Main, Germany correlates with synaptic strength, ${ }^{16-18}$ and special influence was attributed to the nanoscaled diameter of their neck. ${ }^{14}$ In particular, the spine morphology is likely to be important for longterm potentiation (LTP), involved in learning and memory processes. ${ }^{19,20}$ It is therefore important to observe the neurons with a method that is capable of resolving the details of spine shape. ${ }^{12}$ STED microscopy has been successfully used to visualize and analyze dendritic-spine morphology in cell cultures, ${ }^{12,21}$ brain slices, ${ }^{11,14,22,23}$ and even in vivo. ${ }^{24,25}$ Changes in spine morphology after chemical LTP (Refs. 12 and 22) and glutamate uncaging ${ }^{26}$ could be observed, and it could be shown that spine neck plasticity regulates compartmentalization of synapses. ${ }^{14}$ Changes in the actin cytoskeleton of spines were revealed in vivo ${ }^{25}$ and potential correlations between the amplitude of uncaging-evoked excitatory postsynaptic potentials with spine morphology have been investigated. ${ }^{27}$

In addition to noninvasive observation with light microscopy, noninvasive and spatially refined stimulation of biological systems can also be achieved optically due to the development of caged photolyzable components ${ }^{26,28}$ such as neurotansmitters and more recently of optogenetics. ${ }^{29-31}$ Both for optogenetics and uncaging, the light should ideally be delivered to the specimen in a way that allows targeting single excitable units, which can be specific parts of a tissue, single cells, or even subcellular compartments, such as neuronal dendrites or spines. ${ }^{32-34}$ In the case of dendritic spine observation, this is all the more important since it has been shown that dendritic spines exhibit collective 
behaviors even when separated by several microns. ${ }^{35,36}$ Also, nonlinear synaptic integration along dendrites has been found. ${ }^{37,38}$ Therefore, methods to observe the evolution of morphological changes of spines or groups of spines under spatially flexible stimulation have to be devised.

Computer generated holography $(\mathrm{CGH})^{39}$ is efficient both for two-dimensional and three-dimensional light patterning with submicron resolution. ${ }^{32,40}$ Light patterning is achieved by modulating the wavefront of the incident light in the back focal plane of the objective. Phase-only modulation conserves all the incoming laser power and efficiently redistributes light energy in the sample so that no light is wasted. Consequently, CGH enables simultaneous excitation with arbitrary patterns, overcoming the sequential targeting imposed by the use of galvanometric mirrors. ${ }^{41} \mathrm{CGH}$ was used to address dendritic integration on apical dendrites in acute brain slices ${ }^{37,42,43}$ and to control the kinetics of glutamate-evoked currents. ${ }^{40}$

However, holographic stimulation and superresolution imaging have never been combined. Such an optical system would open the possibility for detailed all-optical control and observation of brain circuits, ${ }^{1}$ enabling fundamental discoveries about synapse functioning.

Here, we implement an original optical system for simultaneous holographic photostimulation and superresolution STED imaging. We demonstrate the capabilities of this microscope to precisely monitor morphological changes of dendritic spines after holographic uncaging of glutamate at individually targeted spines.

\section{Results}

\subsection{Optical Setup}

A STED microscope is combined here with CGH for photostimulation of neurons (Fig. 1) and subsequent monitoring of nanoscale morphological changes.

The STED microscope reaches a resolution below $50 \mathrm{~nm}$ and was described in detail earlier. ${ }^{21}$ The excitation beam is derived from a supercontinuum source realized as a photonic crystal fiber (PCF), which generates a broad white-light spectrum from the infrared pulses of the Ti:Sa laser. The excitation wavelength of choice is selected with a band-pass filter. One-photon excitation $(532 \mathrm{~nm}, \sim 1 \mu \mathrm{W})$ is used. A wavelength-tunable depletion beam with a narrow spectrum is generated by wavelength-converting the infrared pulses with an optical parametric oscillator (OPO) [Fig. 1(a)]. Multicolor excitation is then possible in principle. Both stage- and beam-scanning were implemented. Beam-scanning allows keeping the sample stationary relative to the $\mathrm{CGH}$ stimulation pattern. For beam-scanning, a two-axis piezo scanner is used, which tilts a single mirror in two orthogonal axes. Therefore, only a single telescope (not shown in Fig. 1) is required to perfectly conjugate the scanning mirror to the aperture of the objective. A mirror under the objective flips the optical axis from the horizontal to a vertical position before entering the objective as in a usual inverted microscope. Implementing this mirror [DM3 in Fig. 1(a)] as a dichroic mirror allows merging the STED-imaging and the holographic stimulation part with a minimum of optical elements. A 6-mm-thick substrate instead of the common 1-mmthick dichroics is used to ensure that the mirror is not distorted and delivers aberration-free reflections. All light for STED imaging (excitation beam, STED beam, and fluorescence) is reflected by this short-pass dichroic mirror, while the light

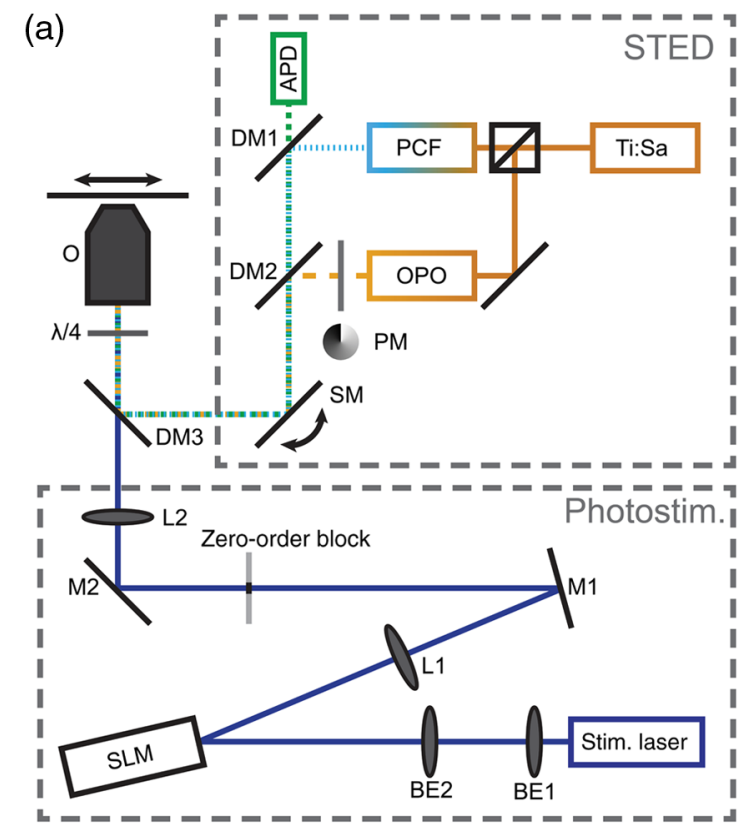

(b)

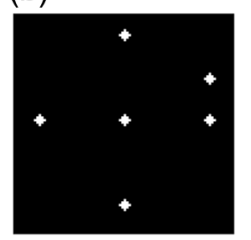

(c)

(e)
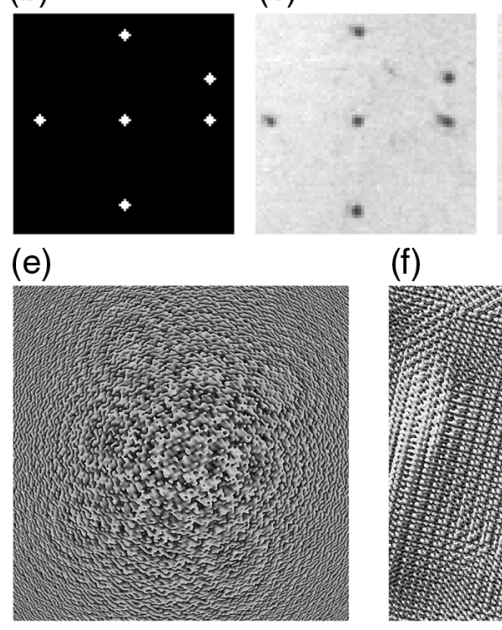

(d)

(f)

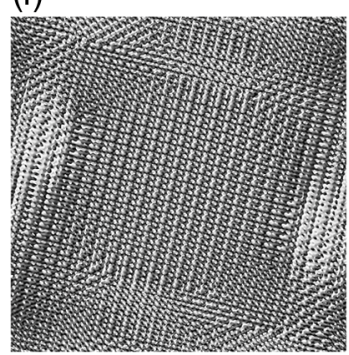

Fig. 1 Optical recording combined with optical control. (a) Schematic optical setup for photostimulation and STED imaging. For STED imaging, both excitation and STED beams were generated from one Ti: Sa laser pumping a PCF and an OPO. Beam paths for excitation, STED, and fluorescence are combined with dichroic mirros DM1 and DM2. A helical phase mask (PM) generated the toroidal STED focus. Fluorescence was recorded with an avalanche photodiode APD. Sample stage-scanning and beam-scanning with a scan mirror (SM) were implemented. For holographic photostimulation, a spatial light modulator (SLM) was imaged into the back focal plane of the objective $\mathrm{O}$ with lenses $\mathrm{L} 1$ (focal length $f=750 \mathrm{~mm}$ ) and L2 $(f=140 \mathrm{~mm})$. Mirror M2 was in a conjugated sample plane for facilitated alignment. Light that is not diffracted by the SLM was blocked in the focal plane of L1 (zero-order block). The laser beam of the stimulation laser (wavelength $403 \mathrm{~nm}$ ) was expanded with the lenses BE1 $(f=10 \mathrm{~mm})$ and BE2 $(f=150 \mathrm{~mm})$. Dichroic mirror DM3 combines imaging and photostimulation beams. (b) through (f) Co-alignment of imaging and holography. (b) A set of diffraction-limited points is holographically generated in the sample plane (points enlarged for display) to bleach holes in a fluorescent layer. (c) The layer is imaged with the scanner that is used for STED imaging showing the bleached holes. (d) Overlay of the images and the intended spot positions. The bleached spots are at the expected positions, confirming correct alignment of the holographic system relative to the imaging. Scale bar is $5 \mu \mathrm{m}$. (e) Hologram that is displayed on the SLM for the spot generation including a Fresnel lens. (f) Without the Fresnel lens, multiple blazed grids are apparent in the hologram. 
for photostimulation $(403 \mathrm{~nm})$ is transmitted. This reflecting configuration for the STED part of the microscope is chosen in order to avoid possible aberrations introduced by transmission through the dichroic mirror. Aberrations introduced in the CGH beam path are less critical as they can be corrected by adding compensating phase profiles to the holograms on the phase modulator (as detailed below). Before reflection on the dichroic mirror, the polarization of the STED beam is vertical, thus maintaining linear polarization after reflection. An achromatic quarter-wave-plate is placed between the dichroic beam splitter and the objective lens so that the STED beam is circularly polarized in the sample plane. Circular polarization is critical to have zero intensity at the center of the depleting donut.

For CGH, a spatial light modulator (SLM) is placed in a plane that is conjugated to the back focal plane of the microscope objective. Usually, the SLM is implemented as a nematic liquid crystal device that allows shifting the phase of the incident light pixel-wise. Full control of the wavefront is thus achieved. An iterative Gerchberg-Saxton algorithm ${ }^{44}$ is commonly used to generate the desired target light distribution in the front focal plane of the objective (the sample plane) by phase modulation.

The SLM is typically illuminated with a collimated laser beam and imaged onto the back focal plane of the objective $\left(\mathrm{BFP}_{\mathrm{O}}\right)$ with a telescope in $4 \mathrm{f}$ configuration. In this case, the SLM is in the object focal plane of the first telescope lens [L1 in Fig. 1(a)]; the distance between the telescope lenses is the sum of their focal lengths $\left(f_{1}+f_{2}\right)$, and the $\mathrm{BFP}_{\mathrm{O}}$ is in the focal plane of the second telescope lens (L2). The total distance between the SLM and the $\mathrm{BFP}_{\mathrm{O}}$ is four focal lengths of the telescope lenses, and hence the name. Then the two main requirements of CGH are fulfilled: on the one hand, projecting the light pattern in the sample plane of the objective and on the other hand, imaging the SLM into the $\mathrm{BFP}_{\mathrm{O}}$ (to relay the phase modulation into the $\mathrm{BFP}_{\mathrm{O}}$ ).

For combination with STED microscopy, the following constraints have to be considered: (1) The objective is a $100 \times$ objective whose back aperture is smaller than the SLM. In addition, a large field of view $(100 \mu \mathrm{m})$ is desired. As a consequence, a demagnification by more than a factor of 5 from the SLM to the $\mathrm{BFP}_{\mathrm{O}}$ was necessary $\left(f_{1} / f_{2}=5.36\right)$. (2) For space constraints, especially the presence of the dichroic mirror for combining the STED beam path with the holography beam path, a minimal distance from the last telescope lens L2 to the objective O has to be met. (3) The total beam path should not be unreasonably long for mechanical stability. Especially problematic can be the distance of the SLM to the first telescope lens L1, because it can become very long in a $4 \mathrm{f}$ system due to the constraints of points 1 and 2. (4) The STED beam path should not be deteriorated by the holography for optimal superresolution imaging. Optical elements that are unnecessary for imaging should therefore be avoided in the STED beam path. In contrast, aberrations in the holography beam path can be corrected with SLM. (5) Light that has not been diffracted by SLM (zero-order) has to be blocked in an intermediate image plane. (6) A mirror in a plane conjugated to the sample plane is desired for alignment purposes.

Therefore, the setup is conceived as a non- $4 \mathrm{f}$ system. This allows increasing the distance between the microscope objective and the lens L2, while decreasing the distance from the SLM to L1. Nevertheless, the SLM is still imaged into the $\mathrm{BFP}_{\mathrm{O}}$. Furthermore, the distance between the two lenses of the telescope, L1 and L2, is chosen larger than the sum of their focal lengths. The zero-order is thus focused before the (intermediate) image plane in the telescope, where it can be easily blocked without introducing a central obstruction in the field of excitation. In order to shift the illumination profiles back into the sample plane, a divergent Fresnel lens is added to the phase profiles of SLM. Since no beam block is needed in the intermediate image plane, a mirror can be placed there for ease of alignment. This allows centering the holographic stimulation beam in the aperture of the objective without shifting the stimulation pattern in the sample plane. By demagnifying the image of the SLM in the back focal plane of the objective $\left(f_{2}>f_{1}\right)$, diffraction angles are increased by the same factor. Therefore, holographic patterns can be projected into a large field of view in the sample plane. We trade a large field of view for some resolution of the holographic patterns (imaging resolution is unaffected), by underfilling the back aperture of the objective. Diffraction-limited holographic spots have a size of $450 \mathrm{~nm}$. This underfilling does not effectively degrade our resolution for uncaging, because diffusion is anyways fast at this spatial scale, giving a stimulation specificity at the micrometer scale. ${ }^{45}$

\subsection{Relative Alignment of Stimulation and STED Imaging}

Photostimulation and imaging must be superposed to enable choosing targets for photostimulation in the fluorescence images. Alignment is performed by bleaching diffraction-limited spots [Fig. 1(b)] with CGH in a fluorescent spin-coated layer that is subsequently imaged [Fig. 1(c)]. Overlaying the observed bleached spots with the intended spot positions confirms the alignment [Fig. 1(d)]. An illustration of the computergenerated hologram displayed on the SLM is shown in Fig. 1(e). It is dominated by the Fresnel lens that is used to axially shift the desired diffracted light pattern from the nondiffracted zero-order light. Rotating the hologram allows correcting by software for a rotation of the field of view of the imaging with respect to SLM. When the hologram is calculated without the Fresnel lens, a superposition of blazed gratings is visible [Fig. 1(f)], as expected for the projection of several points into one plane.

\subsection{Aberration Correction}

The projection of the hologram from the SLM to the $\mathrm{BFP}_{\mathrm{O}}$ traverses the dichroic mirror that combines the STED imaging part and holography part of the setup. Because the holographic beam is, in general, not collimated and the dichroic mirror is thick (to ensure its flatness, see above) and mounted at an angle of $45 \mathrm{deg}$ relative to the holographic beam, aberrations are introduced and have to be corrected by precompensation on the SLM. According phase profiles are therefore added to the holograms. Individual aberrations are represented as Zernike polynomials. ${ }^{46}$ The compensation is adjusted manually under visual control on a camera by projecting diffraction-limited spots on a homogeneous fluorescent layer.

\subsection{Imaging of Dendritic Spines}

The study of induced dynamic changes in spine morphology requires a staining protocol with the following properties: (1) the staining has to label spine morphology in sufficient detail; (2) it has to be live-cell compatible; and (3) there should be a minimal spectral cross-talk between the wavelengths used for stimulation of the neurons and imaging. 

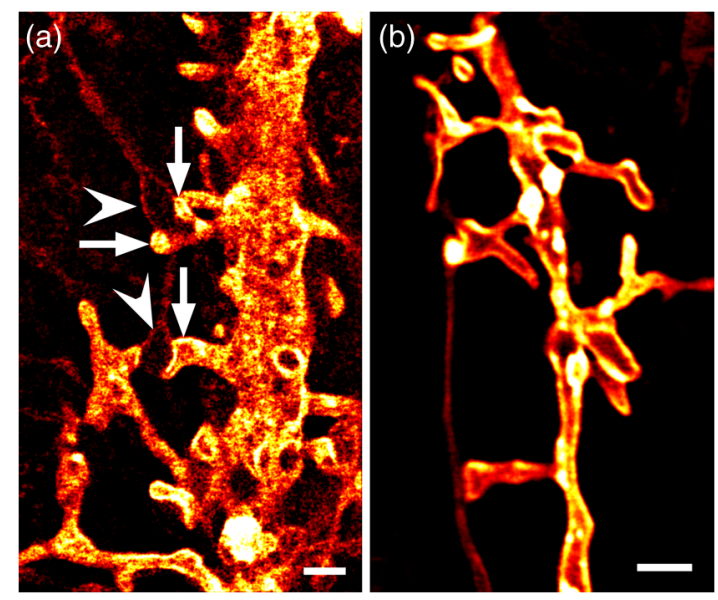

Fig. 2 STED microscopy resolves dendritic spines and synapses. (a) Transfection with YFP-coupled Channelrhodopsin-2 visualizes postsynaptic spines (arrows) and presynaptic boutons (arrowheads) in living neurons. (b) A lipophilic membrane stain with the organic dye Dil depicts a sharp outline of the neuronal structures in living cells. This is especially valuable for small structures like spines. In addition, the axons are labeled so that whole synapses become visible. Scale bars: $1 \mu \mathrm{m}$.

In a first set of experiments, we therefore investigate staining options for primary cultured hippocampal neurons (Fig. 2).

To address point 1 , the resolution of fine details, we seek possibilities for a membrane stain, which delineates better fine morphological details than a cytosolic marker. We demonstrate two solutions: transfection with a fluorescent protein attached to a membrane protein [Fig. 2(a)] or incubation with a synthetic lipophilic dye [Fig. 2(b)], which integrates into the membrane of living cells. Both methods comply with point 2 , the live-cell compatibility.

First, staining via a membrane-bound fluorescent protein is used: expression of YFP-Channelrhodopsin-2 allows STED imaging of dendrites, axons, and synapses in living cells [in Fig. 2(a), some boutons and spines are highlighted with arrowheads and arrows, respectively]. The fluorescent signal reveals the distribution of the light-sensitive ion channel Channelrhodopsin-2 in the dendrite and in spines. This staining strategy should then allow studying correlations among synaptic plasticity, spine morphology, and ion-channel density. An alternative membrane stain for STED imaging consists of lipophilic dyes that integrate into the cell membrane. ${ }^{47}$ In contrast to staining with fluorescent proteins, no transfection or transgenic animal is needed, but the samples can easily be stained at the time of imaging. Here we use DiI, which visualizes synapses because the lipophilic dye stains pre- and postsynapses. Thus, this membrane stain enables resolving the outline of the axon and the postsynaptic dendritic spines in living cells [Fig. 2(b)]. For visualization of synapses, the DiI label has the advantage to stain, in principle, all cells, in contrast to the transfection stain, which depends on the infection rate. Notably, we noticed that cell staining was inhomogeneous with the DiI dye. The wavelengths used for uncaging $(403 \mathrm{~nm})$, fluorescence excitation $(532 \mathrm{~nm})$, and STED beam $(635 \mathrm{~nm})$ are well separated.

In both staining examples, STED microscopy reveals a multitude of protrusions from the dendrites. In cell cultures, many dendrites show the classical form of spines, i.e., with a globular head connected to the dendritic shaft via a constricted region (the neck) [Fig. 3(a)], whereas others show a large variety
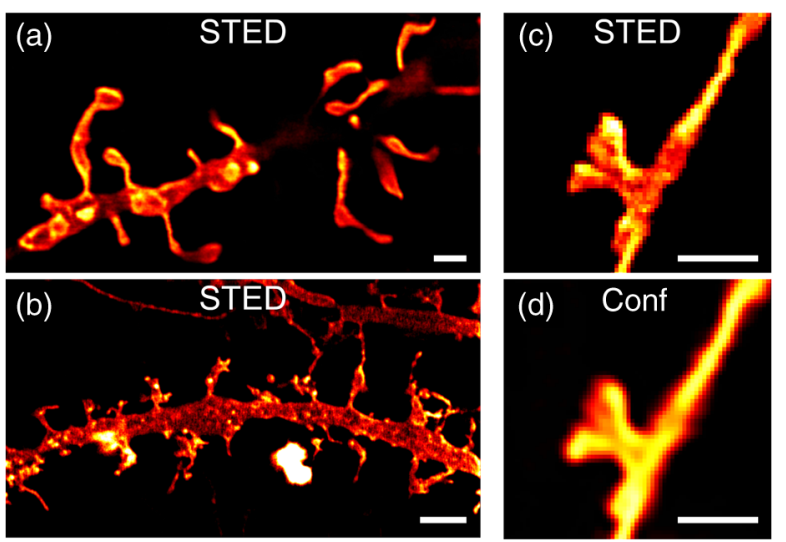

Fig. 3 Spines show a variety of forms that are only resolved with superresolution microscopy. Dendritic spines stained with Dil and visualized in STED microscopy may have the typical form of (a) mushroom spines or (b) more complex shapes. (c) A fork-like spine is resolved in STED imaging, but appears as (d) mushroom spine in confocal microscopy (PSF 190 nm FWHM). Scale bars: $1 \mu \mathrm{m}$ in (a), (c), and (d), and $5 \mu \mathrm{m}$ in (b).

of forms [Fig. 3(b)], which can be mistaken for classical spines if the optical resolution is insufficient. ${ }^{14,48}$ A striking example is shown in Figs. 3(c) and 3(d): a protrusion is resolved by STED microscopy into an unusual form of a fork-like structure with three sub-branches [Fig. 3(c)], but it looks like a spine of the mushroom type at confocal resolution [Fig. 3(d)]. These neurons are cultured cells whose morphology may be different in more physiological environments. However, these images demonstrate the requirement for superresolution imaging when imaging spines and studying synaptic plasticity.

\subsection{Targeted Photostimulation and Superresolution Monitoring}

To evaluate the possibility to stimulate and observe neurons noninvasively, we target selected spines optically with $\mathrm{CGH}$ for stimulation with glutamate uncaging. We observe the morphological changes of single spines with STED microscopy in response to stimulation. To this end, 4-Methoxy-7-nitroindolinyl-caged-L-glutamate (MNI-glutamate) is added to the extracellular solution. MNI-glutamate is an inactive, caged form of the neurotransmitter glutamate, which releases active glutamate when photolysed with $403 \mathrm{~nm}$ light, but not at our imaging wavelengths of 532 and $630 \mathrm{~nm} .{ }^{49}$

First, a synapse is identified [Fig. 4(a), arrow]. The shape of the dendritic spine before glutamate uncaging is recorded [Fig. 4(b), magenta]. Using $\mathrm{CGH}$, glutamate is then released specifically onto this spine to induce synaptic plasticity [Fig. 4(a), inset]. To this end, a train of 40 light pulses of $403 \mathrm{~nm}$ wavelength is delivered at $1 \mathrm{~Hz}$. Each pulse has a length of $2 \mathrm{~ms}$; laser power is $5 \mathrm{~mW}$. Compaction of this spine is seen $25 \mathrm{~min}$ poststimulation [Fig. 4(b), green], suggesting that long-term depression occurred. This spine shrinkage continues and becomes more prominent after 50 min [Fig. 4(c)]. A nonstimulated spine does not change its morphology after 25 min [Fig. 4(d)]. Figures 4(e)-4(j) show the respective overlays separated for clarity.

\section{Summary and Discussion}

We have presented an optical system for noninvasive control and observation of neuronal processes like synaptic plasticity 

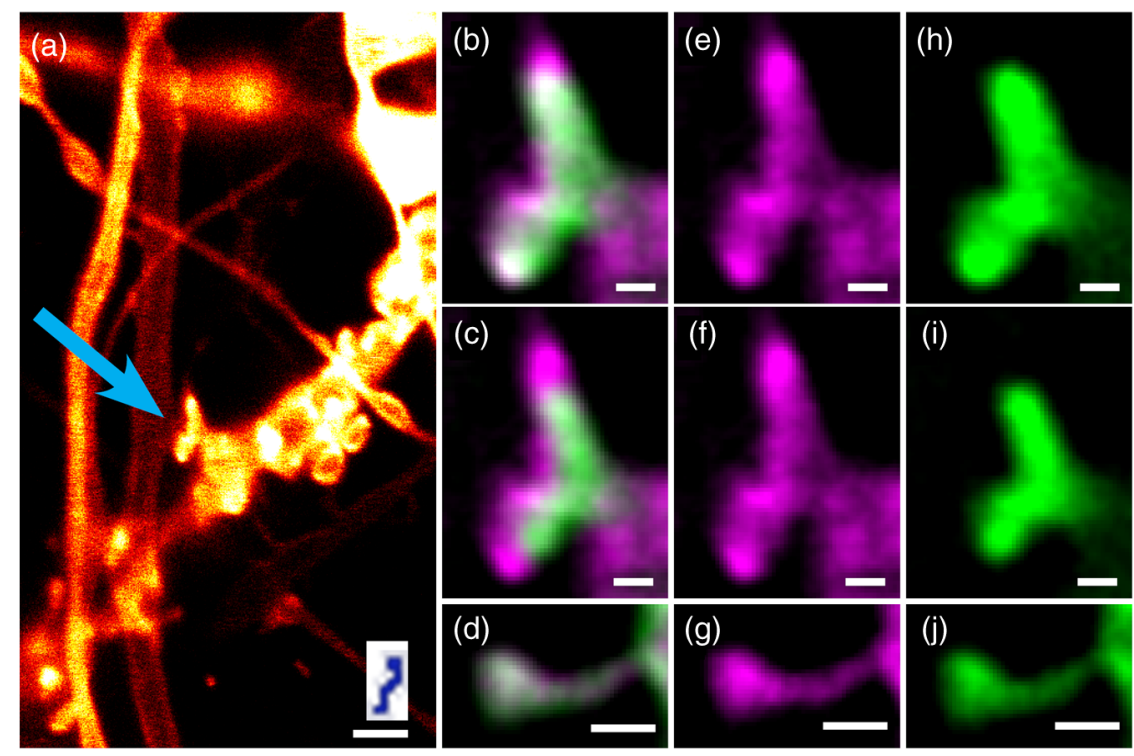

Fig. 4 Optical stimulation of synapses via glutamate uncaging. (a) STED microscopy reveals dendritic spines that can then be targeted with a holographic light pattern (inset). (b) This spine reacts to a glutamate-uncaging protocol; magenta: before uncaging and green: 25 min after uncaging. (c) The effect is stronger after $50 \mathrm{~min}$; magenta: before uncaging and green: after uncaging. (d) Control: nonstimulated spine; magenta: initial image and green: 25 min later. (e) through (j) Separation of overlays for clarity. Scale bars: $1 \mu \mathrm{m}$ in (a) and $0.2 \mu \mathrm{m}$ in (b) through (j).

occurring at a submicron scale. The arrangement is based on STED microscopy for superresolution imaging and CGH for precise optical stimulation. These techniques are combined with a membrane stain via lipophilic dyes for sharp visualization of neurites and with caged neurotransmitters ${ }^{50}$ for enabling the light-induced stimulation of neurons via natural pathways. The system is tested using cell cultures, but both STED microscopy and CGH perform well in more physiological contexts like brain slices $^{22,40}$ and even in vivo. ${ }^{24,51}$ In slices, STED imaging can reach a depth of at least $120 \mu \mathrm{m} .{ }^{22}$ We have shown here that STED imaging of spines marked with a lipophilic membrane dye is possible over $50 \mathrm{~min}$ (typically about one image every $10 \mathrm{~min}$ ). The possibility of repeated STED imaging of neurons has also been confirmed in other studies, for spines ${ }^{14,24,23}$ and axons. ${ }^{7}$ Both STED microscopy ${ }^{23}$ and CGH can use two-photon excitation. ${ }^{52}$ The use of temporal focusing for $\mathrm{CGH}$ ensures the confinement of uncaging also for laterally extended light patterns. $^{52}$

CGH is integrated in a home-built STED microscope according to an original design for relaying the SLM into the back focal plane of the objective (Fig. 1). Staining neurons with membrane-targeted fluorescent proteins or lipophilic membrane dyes is tested and allows the visualization of details of dendritic spines and synapses. Pre- and postsynaptic membranes are stained (Fig. 2), which enables the visualization of complete synapses. Noticeably, not all cells are stained by the lipophilic dye DiI with the same density, which is in contrast with what is commonly reported in the literature. ${ }^{53,54}$

Comparison between STED and confocal images confirms that spines may easily be assigned to a wrong class, ${ }^{14}$ when the optical resolution is insufficient (Fig. 3). Membrane staining better highlights the difference in resolution than cell-filling stains as it delineates sharper morphological details. Membrane staining is thus expected to be more precise about the dendrite structure.
It is finally shown that the combination STED-CGH can be used for optical stimulation of neurons and simultaneous noninvasive detailed optical observation (Fig. 4).

Alternatively to $\mathrm{CGH}$, light for uncaging of neurotransmitters can be controlled with galvanometric mirrors and other point-scanning methods. In contrast to scanning techniques, CGH has the advantage to allow simultaneous excitation with arbitrary shapes. ${ }^{41}$ Since light can be applied with CGH to several neurons, dendrites, axons, or spines, studying the interplay of simultaneous multisite stimulation becomes possible. This will open the route for examining how synapses change their morphology in response to neuronal-network activity, particularly in the case of nonlinear dendritic integration. ${ }^{37}$ The approach of optically stimulating cells and observing reactions at a subcellular scale is not limited to the release of neurotransmitters. The combination with optogenetics for control of lightgated ion channels $\mathrm{s}^{2,55,51}$ or other light-sensitive effector molecules such as G-proteins ${ }^{30}$ is equally possible. Therefore, these all-optical methods for cell stimulation and monitoring are expected to spread to various fields of biological research in neuroscience and beyond.

\section{Methods}

\subsection{Sample Preparation}

Primary neuronal cultures were prepared as described in Ref. 21 from mouse embryos at embryonic day 16 and cultured in gliaconditioned medium. Neurons were plated at 40,000 cells per coverslip (\#1, BK-7, diameter $18 \mathrm{~mm}$, Marienfeld Superior, Menzel-Gläser GmbH, Braunschweig, Germany) in glia-conditioned medium and used at day in vitro (DIV) 18 to 20 .

Mice were treated in accordance with European directives (86/609/EEC and 2010-63-EU) and the French legislation. Experiments were approved by the ethics committee of the University Paris Descartes (Permit Number: CEEA34.FD.047.11). 
Samples were stained by incubation in Vybrant DiI (V22885, Life Technologies, Saint Aubin, France) in a 1:400 $(2.5 \mu \mathrm{l} / \mathrm{ml})$ dilution $(2.5 \mu \mathrm{g} / \mathrm{ml}$ final DiI concentration $)$ in neurobasal medium for 15 to $20 \mathrm{~min}$, at $37^{\circ} \mathrm{C}, 5 \% \mathrm{CO}_{2}$, followed by 15 to 20 min washing in neurobasal medium at $37^{\circ} \mathrm{C}, 5 \% \mathrm{CO}_{2}$. Microscopy was then performed at room temperature in Hepesbuffered artificial cerebrospinal fluid (ACSF, in mM: $125 \mathrm{NaCl}$, $2.5 \mathrm{KCl}, 25 \mathrm{Hepes}, 2 \mathrm{CaCl}_{2}, 1.3 \mathrm{MgCl}_{2} 33$ Glucose; $\mathrm{pH}$ 7.4). For glutamate-uncaging experiments, MNI-glutamate $(0.2 \mathrm{mM})$ and Forskolin $(50 \mu \mathrm{M})$ were added and $\mathrm{MgCl}_{2}$ was omitted. ${ }^{26}$

For the Channelrhodopsin-YFP staining, neurons were transfected with AAV2/5.hSynapsin.hChR2(H134R).EYFP. WPREhGH $\left(2.1 * 10^{10} \mathrm{GC} / \mathrm{mL}\right.$, UPenn vector core, Philadelphia, Pennsylvania) at DIV 12 and imaged 10 days later at room temperature in ACSF.

\subsection{STED Microscopy}

A home-built STED microscope was used that derives excitation and STED beams from a single laser pump (Fig. 1), reaching a resolution below $50 \mathrm{~nm}$; details have been described elsewhere. ${ }^{21}$ The resolution was determined by imaging small structures and measuring the apparent width (full width at halfmaximum). The STED wavelength was $635 \mathrm{~nm}(590 \mathrm{~nm}$ for YFP images). Fluorescence excitation was derived from a PCF (femtoWHITE-800, NKT Photonics A/S, Birkerød, Denmark); a dielectric filter (532/10, Chroma, Bellows Falls, Vermont) was used for selecting the excitation wavelength. Fluorescence was detected by an avalanche photodiode (SPCM-AQRH13, Perkin Elmer, Fremont, California) behind a 585/65 filter (Chroma) (525/50 for YFP images). A helical phase mask of charge one (VPP-A1, RPC Photonics, New York) generated the toroidal STED focus. For STED imaging, a pixel size of $20 \mathrm{~nm}$ was used with a pixel dwell time of $50 \mu \mathrm{s}$. Excitation powers were $\sim 1 \mu \mathrm{W}$; STED power was $\sim 10 \mathrm{~mW}(1.7 \mu \mathrm{W}$ and $11 \mathrm{~mW}$ for the YFP images). For stage-scanning, a piezo stage P734.2CL and digital controller E710 (Physik Instrumente, Karlsruhe, Germany) were used. A two-axes piezoelectric scanner (S-330.8SL with controller E505, Physik Instrumente) allowed alternatively beam-scanning.

Images were linearly deconvolved (Wiener filtered) for display.

\subsection{Digital Holography}

Phase holograms that were displayed on the SLM were calculated using an iterative Fourier transform algorithm ${ }^{44}$ with Wavefront Designer IV, an in-house software written in C++. They were displayed on a PALSLM (PS00830 with PPM driver, Hamamatsu, Massy, France). Uncaging regions were chosen in overview images that were recorded with the STED microscope operating in confocal mode.

The uncaging laser was a cw diode laser (Cube 1069413/AH, Coherent, $403 \mathrm{~nm}, 50 \mathrm{~mW}$ ). Power for uncaging was measured in front of the last dichroic mirror (DM3 in Fig. 1) and set to $5 \mathrm{~mW}$. Power was adjusted with a half-wave-plate and a polarizing beam splitter. Laser emission was controlled via TTL signals to the trigger input of the laser. Pulse trains ( 40 pulses, $2 \mathrm{~ms}$ each, $1 \mathrm{~Hz}$ ) were generated with custom-written LabView routines and an NIDAQ card (National Instruments, Austin, Texas). In addition, the laser was mechanically shuttered before and after pulse trains to avoid any light emission due to limited contrast ratio.

\subsection{Fluorescent Layers}

Fluorescent layers were made of Rhodamine $6 \mathrm{G}$ in polymethylmethacrylate (PMMA): $20 \mu \mathrm{l}$ of a Rhodamine $6 \mathrm{G}$ solution (1M Rhodamine in a 2\% w/v PMMA-in-chloroform solution) was spin-coated onto coverslips (\#1, $25 \mathrm{~mm}$ BK-7, Marienfeld Superior, Menzel-Gläser GmbH, Braunschweig, Germany) at $8500 \mathrm{rpm}$.

\section{Acknowledgments}

We thank Isabelle Fanget, Asma Soltani, and Solène Lebrun for preparation of neuronal cultures, Christophe Tourain for custom-built electronics, Patrice Jegouzo for custom-built mechanical parts, and Vincent de Sars for help with Wavefront Designer IV. We are thankful to Olivier Stettler for valuable discussions and to Rossella Conti for help in preliminary experiments. This work received financial support from the network Ecole des Neurosciences de Paris and Agence Nationale de la Recherche (ANR-10-INBS-04, France-BioImaging Infrastructure network) and from France Alzheimer and the Agence Nationale pour la Recherche (ANR-12-Polybex). M.A.L. received funding from the People Programme (Marie Curie Actions) of the European Union's Seventh Framework Programme (FP7/2007-2013) under REA Grant Agreement No. PIEF-GA-2011-297917.

\section{References}

1. V. Emiliani et al., "All-optical interrogation of neural circuits," J. Neurosci. 35(41), 13917-13926 (2015).

2. S. W. Hell, "Far-field optical nanoscopy," Science 316(5828), 11531158 (2007).

3. M. A. Lauterbach, "Finding, defining and breaking the diffraction barrier in microscopy—a historical perspective," Opt. Nanosc. 1(1), 8 (2012).

4. S. W. Hell and J. Wichmann, "Breaking the diffraction resolution limit by stimulated emission: stimulated-emission-depletion fluorescence microscopy," Opt. Lett. 19(11), 780-782 (1994).

5. E. Rittweger et al., "STED microscopy reveals crystal colour centres with nanometric resolution," Nat. Photonics 3(3), 144-147 (2009).

6. V. Westphal and S. W. Hell, "Nanoscale resolution in the focal plane of an optical microscope," Phys. Rev. Lett. 94(14), 143903 (2005).

7. V. Westphal et al., "Video-rate far-field optical nanoscopy dissects synaptic vesicle movement," Science 320(5873), 246-249 (2008).

8. M. A. Lauterbach et al., "Dynamic imaging of colloidal-crystal nanostructurs at 200 frames per second," Langmuir 26(18), 14400-14404 (2010).

9. V. Westphal et al., "Dynamic far-field fluorescence nanoscopy," New J. Phys. 9, 435 (2007).

10. J. Schneider et al., "Ultrafast, temporally stochastic STED nanoscopy of millisecond dynamics," Nat. Methods 12(9), 827-830 (2015).

11. L. S. Wijetunge et al., "Stimulated emission depletion (STED) microscopy reveals nanoscale defects in the developmental trajectory of dendritic spine morphogenesis in a mouse model of fragile X syndrome," J. Neurosci. 34(18), 6405-6412 (2014).

12. U. V. Nägerl et al., "Live-cell imaging of dendritic spines by STED microscopy," Proc. Natl. Acad. Sci. 105(48), 18982-18987 (2008).

13. R. Yuste and T. Bonhoeffer, "Genesis of dendritic spines: insights from ultrastructural and imaging studies," Nat. Rev. Neurosci. 5(1), 24-34 (2004).

14. J. Tønnesen et al., "Spine neck plasticity regulates compartmentalization of synapses," Nat. Neurosci. 17(5), 678-685 (2014).

15. V. A. Alvarez and B. L. Sabatini, "Anatomical and physiological plasticity of dendritic spines," Аnnu. Rev. Neurosci. 30, 79-97 (2007).

16. C. Lang et al., "Transient expansion of synaptically connected dendritic spines upon induction of hippocampal long-term potentiation," Proc. Natl. Acad. Sci. 101(47), 16665-16670 (2004).

17. M. Matsuzaki et al., "Structural basis of long-term potentiation in single dendritic spines," Nature 429(6993), 761-766 (2004). 
18. U. V. Nägerl et al., "Bidirectional activity-dependent morphological plasticity in hippocampal neurons," Neuron 44(5), 759-767 (2004).

19. M. Matsuzaki et al., "Dendritic spine geometry is critical for AMPA receptor expression in hippocampal CA1 pyramidal neurons," Nat. Neurosci. 4(11), 1086-1092 (2001).

20. Z. Nusser et al., "Cell type and pathway dependence of synaptic AMPA receptor number and variability in the hippocampus," Neuron 21(3), 545-559 (1998).

21. M. A Lauterbach et al., "STED microscope with spiral phase contrast," Sci. Rep. 3, 2050 (2013).

22. N. T. Urban et al., "STED nanoscopy of actin dynamics in synapses deep inside living brain slices," Biophys. J. 101(5), 1277-1284 (2011).

23. J. B. Ding, K. T. Takasaki, and B. L. Sabatini, "Supraresolution imaging in brain slices using stimulated-emission depletion two-photon laser scanning microscopy," Neuron 63(4), 429-437 (2009).

24. S. Berning et al., "Nanoscopy in a living mouse brain," Science 335, 551 (2012).

25. K. I. Willig et al., "Nanoscopy of filamentous actin in cortical dendrites of a living mouse," Biophys. J. 106(1), L01-L03 (2014).

26. A. Govindarajan et al., "The dendritic branch is the preferred integrative unit for protein synthesis-dependent LTP," Neuron 69(1), 132-146 (2011).

27. K. Takasaki and B. L. Sabatini, "Super-resolution 2-photon microscopy reveals that the morphology of each dendritic spine correlates with diffusive but not synaptic properties," Front Neuroanat. 8, 29 (2014).

28. H.-B. Kwon and B. L. Sabatini, "Glutamate induces de novo growth of functional spines in developing cortex," Nature 474(7349), 100-104 (2011).

29. G. Nagel et al., "Channelrhodopsin-1: a light-gated proton channel in green algae," Science 296(5577), 2395-2398 (2002).

30. R. D. Airan et al., "Temporally precise in vivo control of intracellular signalling," Nature 458(7241), 1025-1029 (2009).

31. A. Hayashi-Takagi et al., "Labelling and optical erasure of synaptic memory traces in the motor cortex," Nature 525(7569), 333-338 (2015).

32. E. Papagiakoumou, "Optical developments for optogenetics," Biol. Cell 105(10), 443-464 (2013).

33. D. Oron et al., "Two-photon optogenetics," Prog. Brain Res. 196, 119-143 (2012)

34. A. M. Packer et al., "Two-photon optogenetics of dendritic spines and neural circuits," Nat. Methods 9(12), 1202-1205 (2012).

35. C. D. Harvey and K. Svoboda, "Locally dynamic synaptic learning rules in pyramidal neuron dendrites," Nature 450(7173), 1195-1200 (2007).

36. W. C. Oh, L. K. Parajuli, and K. Zito, "Heterosynaptic structural plasticity on local dendritic segments of hippocampal CA1 neurons," Cell Rep. 10(2), 162-169 (2015).

37. S. Yang et al., "Three-dimensional holographic photostimulation of the dendritic arbor," J. Neural Eng. 8, 046002 (2011).

38. G. Major, M. E. Larkum, and J. Schiller, "Active properties of neocortical pyramidal neuron dendrites," Аnпи. Rev. Neurosci. 36(1), 1-24 (2013).

39. M. Reicherter et al., "Optical particle trapping with computer-generated holograms written on a liquid-crystal display," Opt. Lett. 24(9), 608-610 (1999).

40. C. Lutz et al., "Holographic photolysis of caged neurotransmitters," Nat. Methods 5(9), 821-827 (2008).

41. A. Vaziri and V. Emiliani, "Reshaping the optical dimension in optogenetics," Curr. Opin. Neurobiol. 22(1), 128-137 (2012).

42. F. Anselmi et al., "Three-dimensional imaging and photostimulation by remote-focusing and holographic light patterning," Proc. Natl. Acad. Sci. 108(49), 19504-19509 (2011).

43. S. Yang, V. Emiliani, and C.-M. Tang, "The kinetics of multibranch integration on the dendritic arbor of CA1 pyramidal neurons," Front Cell Neurosci. 8, 127 (2014).

44. R. W. Gerchberg and W. O. Saxton, "A practical algorithm for the determination of phase from image and diffraction plane pictures," Optik 35(2), 237-246 (1972).

45. D. A. DiGregorio et al., "Desensitization properties of AMPA receptors at the cerebellar mossy fiber granule cell synapse," J. Neurosci. 27(31), 8344-8357 (2007).

46. F. Zernike, "Beugungstheorie des Schneidenverfahrens und seiner verbesserten Form, der Phasenkontrastmethode," Physica 1, 689-704 (1934).
47. E. Wagner et al., "Stimulated emission depletion live-cell super-resolution imaging shows proliferative remodeling of t-tubule membrane structures after myocardial infarction," Circ. Res. 111(4), 402-414 (2012).

48. A. Attardo, J. E. Fitzgerald, and M. J. Schnitzer, "Impermanence of dendritic spines in live adult CA1 hippocampus," Nature 523(7562), 592-596 (2015).

49. F. F. Trigo, J. E T. Corrie, and D. Ogden, "Laser photolysis of caged compounds at $405 \mathrm{~nm}$ : photochemical advantages, localisation, phototoxicity and methods for calibration," J. Neurosci. Methods 180(1), 9-21 (2009).

50. C.-M. Tang, Photolysis of Caged Neurotransmitters: Theory and Procedures for Light Delivery, Vol. 37, pp. 6.21.1-6.21.12, John Wiley \& Sons Inc., Hoboken, New Jersey (2006).

51. V. Szabo et al., "Spatially selective holographic photoactivation and functional fluorescence imaging in freely behaving mice with a fiberscope," Neuron 84(6), 1157-1169 (2014).

52. E. Papagiakoumou et al., "Patterned two-photon illumination by spatiotemporal shaping of ultrashort pulses," Opt. Express 16(26), 22039-22047 (2008).

53. M. G. Honig and R. I. Hume, "Fluorescent carbocyanine dyes allow living neurons of identified origin to be studied in long-term cultures," J. Cell Biol. 103(1), 171-187 (1986).

54. M. G. Honig and R. I. Hume, "DiI and DiO: versatile fluorescent dyes for neuronal labelling and pathway tracing," Trends Neurosci. 12(9), 333-341 (1989).

55. O. Yizhar et al., "Optogenetics in neural systems," Neuron 71(1), 9-34 (2011).

Marcel Andreas Lauterbach studied physics in Heidelberg, Germany, and Tucson, Arizona. After his diploma thesis about adaptive optics in the lab of Winfried Denk, he did his PhD about STED microscopy with Stefan Hell in Göttingen, Germany. He then worked as a postdoc in the lab of Valentina Emiliani in Paris, France, on applications of STED microscopy, computer generated holography, and HiLo microscopy. Currently, he is working in the lab of Gilles Laurent in Frankfurt, Germany, on advanced imaging methods for neuroscience.

Marc Guillon studied physics at École Normale Supérieure de Cachan, France. After a PhD at Haute-Provence observatory, France, and a postdoc in Dundee, Scotland, in optomechanics, he joined the group of Valentina Emiliani as an assistant professor to develop superresolution STED microscopy at University Paris Descartes, where he received his venia legendi. His main interests include computer generated holography, superresolution, and phase contrast microscopy as well as singular optics.

Claire Desnos studied cell physiology at the Université de Tours, France. After getting a PhD studying the vesicular monoamine transporter at the Institut de Biologie Physico-Chimique, Paris, and a postdoc in the University of California San Francisco on synaptic vesicle biogenesis, she obtained a permanent position at the Centre National de la Recherche Scientifique. Now in the group of François Darchen, her research focuses on the traffic of secretory vesicles and synaptic plasticity in Alzheimer's disease.

Dany Khamsing studied biology at the University of Paris Descartes, France. After her master degree in cellular biology, physiology, and pathology, she started her thesis about the synaptic protein synthesis and the physiopathology of Alzheimer's disease in the Neurophotonics Laboratory of Valentina Emiliani in Paris, France.

Zahra Jaffal studied biology/biochemistry in Beirut, Lebanon, and Paris, France. After her master degree in "Structure et interaction des macromolécules génomique et fonctionnelle," she did her $\mathrm{PhD}$ about the deregulation of protein synthesis and mTOR pathway in Alzheimer's disease in the lab of François Darchen in Paris, France.

François Darchen studied medicine and biology in Paris, France. He did his PhD on the uptake of neurotransmitters in secretory vesicles and the secretory process. He pursued his work on exocytosis of secretory vesicles as a postdoc in Jean-Pierre Henry's lab and then as a group leader in Paris. He is currently working on the 
pathophysiology of Alzheimer's disease and autism as a group leader in Paris, France.

Valentina Emiliani is the director of the Neurophotonics Laboratory. She obtained her PhD in physics at the University La Sapienza Rome. As a postdoc, she investigated carrier transport in quantum wires
(Max Born Institut, Berlin) and light propagation in disordered structures (LENS, Florence) by SNOM and cell mechanotransduction by optical tweezers (Institute Jacques Monod, Paris). In 2005, she formed the Wavefront Engineering Microscopy group at Paris Descartes University, pioneering the use of wavefront shaping for neuroscience. 